\title{
À PROPOS DU TOUR DU MONDE EN BALLON
}

\author{
Georges Dhonneur(1), Thiéry Beaudenon ${ }^{(2)}$, Didier Renaut ${ }^{(3)}$ et \\ Luc Trullemans ${ }^{(4)}$ \\ (1) Société météorologique de France \\ 1, quai Branly \\ 75340 Paris Cedex 07 \\ (2) Météo-France, Centre départemental de la météorologie de la Marne, \\ Reims \\ (3) Météo-France, Service central de la communication \\ et de la commercialisation, Paris \\ (4) Institut royal météorologique, Bruxelles, BELGIQUE
}

\section{RÉSUMÉ}

Cet article s'appuie sur l'exploit récent des aérostiers Bertrand Piccard et Brian Jones pour rappeler le principe de fonctionnement des divers types de ballons, expliquer comment la conduite d'un long voyage en ballon utilise les courants-jets et détailler l'assistance météorologique mise en œuvre lors du tour du monde.

\section{ABSTRACT About around-the-world balloon travel \\ Based on the recent achievement of the balloonists Bertrand Piccard and Brian Jones, this article describes how the various kinds of balloon operate, explains how a long flight uses the jet-streams, and details the meteorologi- cal assistance given during the journey around the world.}

Le 20 mars 1999, à 9 h 54 UTC, les aérostiers Bertrand Piccard (Suisse) et Brian Jones (Royaume-Uni) ont bouclé au-dessus de la Mauritanie le premier tour du monde sans escale en ballon, après avoir parcouru 42810 kilomètres en 19 jours, 1 heure et 49 minutes. Leur périple s'est en fait achevé le lendemain lorsque leur ballon, le Breitling Orbiter III, s'est posé en Égypte à 6 h 03 UTC ; il avait commencé dans la petite ville de Château d'Oex, en Suisse, lors du décollage du ballon, le $1^{\text {er }}$ mars à $8 \mathrm{~h} 05$ UTC.

Quelques chiffres permettent de mieux situer la nature de cet exploit sans précédent : la vitesse moyenne du Breitling Orbiter III a été de $97 \mathrm{~km} / \mathrm{h}$ (en comptant le parcours jusqu'à l'Égypte). La vitesse maximale $(240 \mathrm{~km} / \mathrm{h})$ a été atteinte le 20 mars ; au contraire, entre le 14 et le 15 mars, le ballon a volé très lentement, à $9000 \mathrm{~m}$ d'altitude au sud d'Hawaii. L'altitude la plus élevée a été supérieure à $11500 \mathrm{~m}$ (le 20 mars) et la plus basse, en dehors des phases de décollage et d'atterrissage, d'environ $3500 \mathrm{~m}$ (le 7 mars).

Cette fabuleuse aventure a résulté de la conjonction de performances exceptionnelles sur les plans humain, technologique et scientifique. 


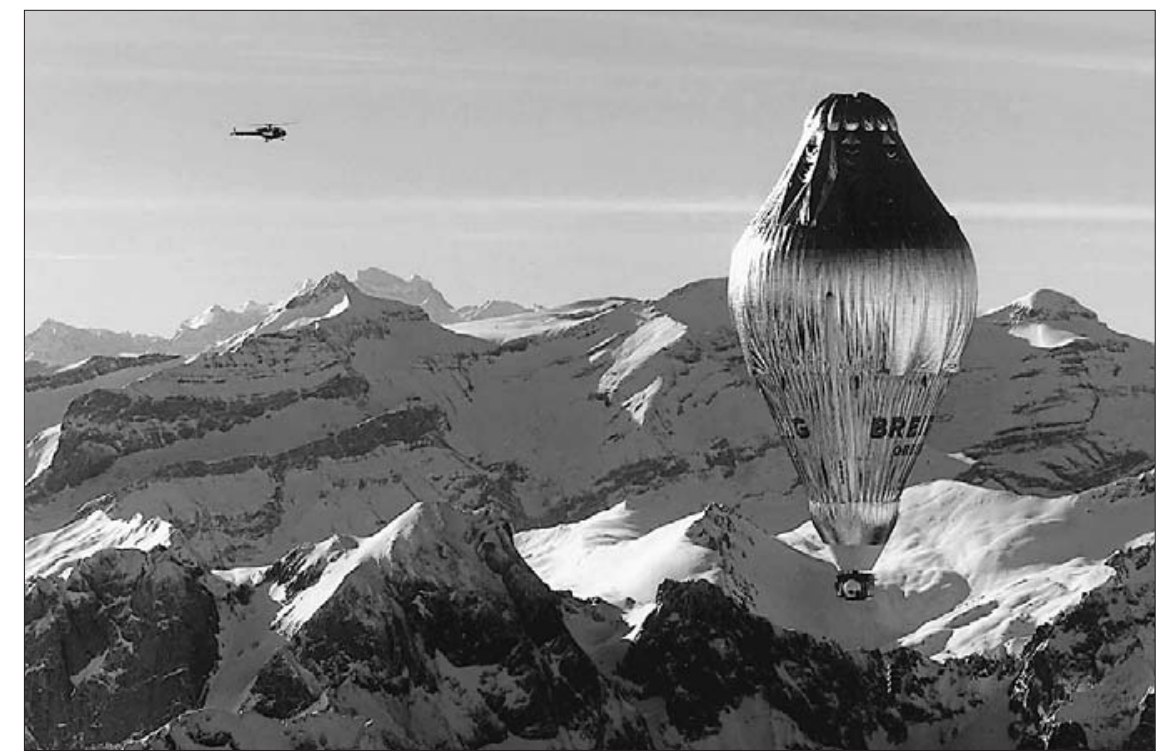

Le Breitling Orbiter III au-dessus des Alpes suisses, juste après son décollage de Château d'Oex, le $1^{\text {er }}$ mars 1999. (@ AFP Photo, Fabrice Coffrini)

\section{LES HOMMES}

Bertrand Piccard

Né en 1958, marié et père de trois filles, Bertrand Piccard est docteur en médecine et spécialisé en psychiatrie. Il a toujours eu la passion du vol libre : deltaplane, ULM et parapente n'ont plus de secrets pour lui. Il effectue en 1979 son premier vol en montgolfière. En 1997 et en 1998, il a déjà tenté par deux fois de faire le tour du monde en ballon, sans succès, en compagnie de Wim Verstraeten (Belgique).

La fibre exploratrice et aventurière qui a poussé Bertrand Piccard à cette prouesse n'est sans doute pas sans rapport avec les talents de la lignée de savants-explorateurs dont il est issu.

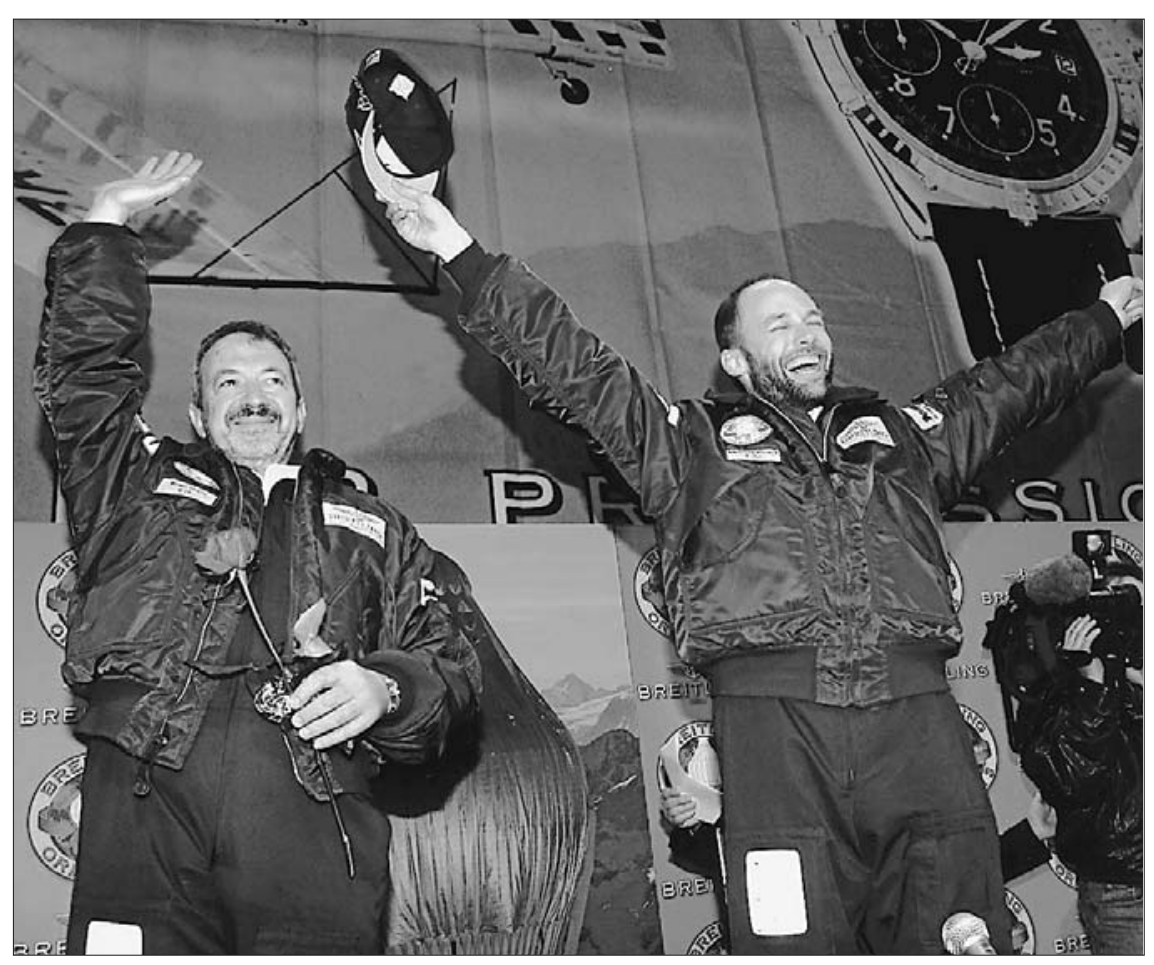

Les aérostiers Brian Jones (à gauche) et Bertrand Piccard (à droite) saluent le public après leur succès, lors de la conférence de presse du 22 mars 1999, à Genève, au lendemain de leur atterrissage en Égypte. (@ AFP Photo, Philippe Desmazes) 
Les Piccard, de savants aventuriers ou des aventuriers savants

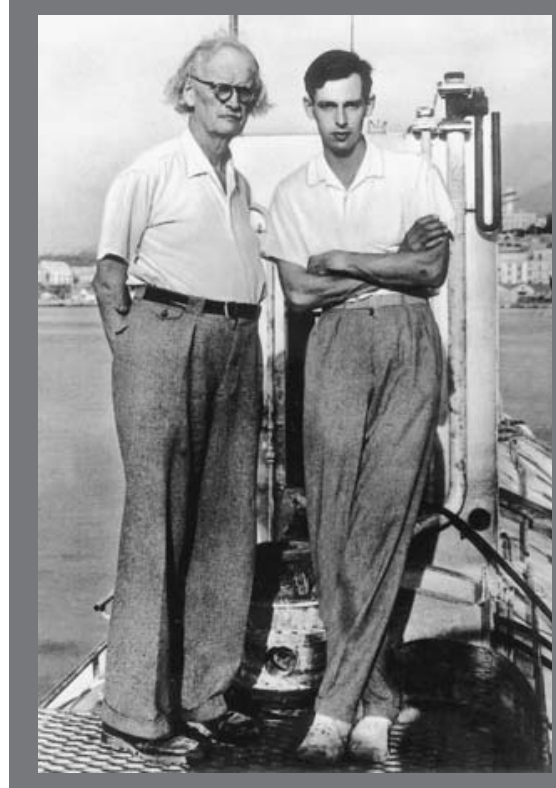

Auguste Piccard (à gauche) et son fils Jacques sur le pont du bathyscaphe Trieste, vers 1960. (C) AFP Photo) mandes du bathyscaphe ; il battra le 23 janvier 1960 le record du monde de profondeur de plongée dans la fosse des Mariannes (océan Pacifique) en descendant jusqu'à 10916 mètres, ce qui représente une pression de 1057 atmosphères.

Bertrand Piccard est le fils de Jacques.
Brian Jones

Les routeurs météo : Luc Trullemans et Pierre Eckert
Né en 1947, marié, père de deux enfants et grand-père de trois petits-enfants, Brian Jones est d'abord un aviateur. Il a passé treize ans au service de la Royal Air Force britannique. Après avoir découvert les joies de l'aérostat en 1986, il est devenu instructeur pour le vol en ballon en 1989. Pilote de réserve lors des tentatives précédentes de Bertrand Piccard, il a subi tous les entraînements nécessaires. Jusqu'au vol qui a conduit à l'exploit, il était directeur du projet Breitling Orbiter III.

Même si la postérité retiendra surtout le nom des deux aérostiers, ce record du monde est aussi le fruit du travail acharné de toute une équipe. Pour ce qui est de l'assistance météorologique au vol, il nous faut dire quelques mots des deux météorologistes « routeurs » qui ont eu la lourde charge de diriger, depuis le sol, le ballon et ses pilotes en fonction des vents observés et prévus dans la haute troposphère.

Luc Trullemans est météorologiste à l'Institut royal météorologique (IRM) de Belgique. Il s'est fait connaître en participant à la mise au point d'un modèle de transport atmosphérique des «nuages » radioactifs, suite à la catastrophe de Tchernobyl. C'est un modèle dérivé de celui-ci que Luc Trullemans a employé pour prévoir les trajectoires successives du Breitling Orbiter III. Lors du récent Festival international de météo, qui s'est tenu à Québec du 15 au 18 avril derniers, Luc Trullemans a remporté le «prix des scientifiques » pour ses présentations du bulletin météo sur la chaîne de télévision RTL-TVI.

Pierre Eckert est météorologiste à l'Institut suisse de météorologie (ISM), après avoir été physicien nucléaire au $\mathrm{CERN}^{(1)}$ à Genève. Pour l'assistance météorologique au Breitling Orbiter III, Luc Trullemans et Pierre Eckert ont travaillé en tandem au centre de contrôle de Genève-Cointrin. Le premier utilisait les prévisions numériques du modèle de la National Oceanic and Atmospheric Administration (NOAA, États-Unis), tandis que le second se servait de celles du modèle du Centre européen pour les prévisions météorologiques à moyen terme (CEPMMT).

(1) Organisation européenne pour la recherche nucléaire (initialement Conseil européen pour la recherche nucléaire). 
Luc Trullemans (à gauche) et Pierre Eckert en train d'expliquer le courant-jet subtropical au centre de contrôle de Genève-Cointrin, le 14 mars 1999.

(๔ AFP Photo, Martial Trezzini)

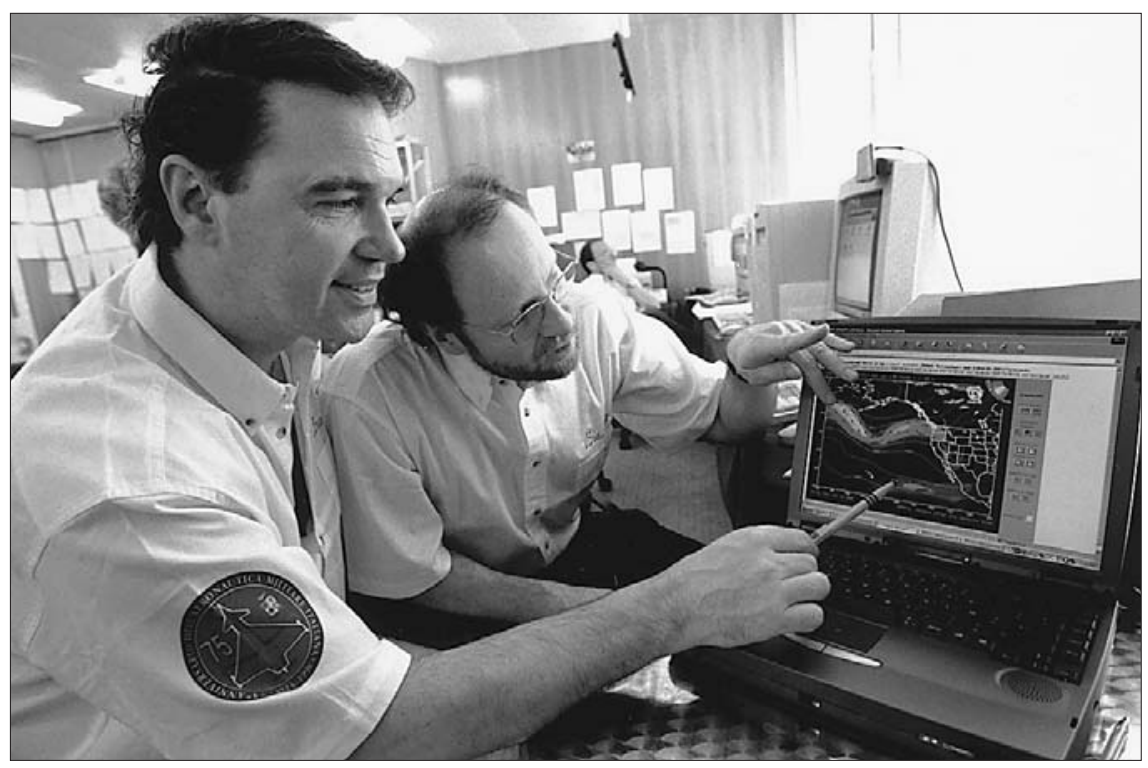

LA TECHNIQUE

Le ballon
Le Breitling Orbiter III est une machine gigantesque : 55 mètres de haut, un poids de 8,1 tonnes et un ballon pouvant contenir $18500 \mathrm{~m}^{3}$ d'hélium.

Le ballon du Breitling Orbiter III est un ballon mixte, ou ballon de type Rozier, qui combine le principe de la montgolfière et celui du ballon à gaz. Il comprend une poche d'hélium disposée à l'intérieur d'une enveloppe à air chaud (figure 1). De jour, les rayons du soleil chauffent la poche d'hélium qui se dilate. Le ballon a tendance à monter. Si l'effet de dilatation est trop important, il faut libérer de l'hélium pour freiner l'ascension. De nuit, au contraire, l'hélium se refroidit et se contracte ; le ballon a tendance à descendre et c'est la mise en marche des brûleurs de l'enveloppe à air chaud qui permet de stabiliser son altitude.

\section{Le ballon à gaz, la montgolfière et le dirigeable}

Le ballon à gaz, la montgolfière et le dirigeable sont des aérostats (ou plus légers que l'air). Le dirigeable est équipé d'une propulsion motorisée. Son enveloppe rigide renferme un gaz plus léger que l'air, l'hélium (gaz ininflammable). Dans un passé encore récent, on employait l'hydrogène (gaz inflammable). Muni d'une hélice, le dirigeable se déplace dans les airs comme un sous-marin dans les mers. Il peut voler pratiquement par tous les temps.

Le ballon à gaz possède une enveloppe constituée de plusieurs fuseaux de tissu caoutchouté. Il peut voler en conditions météorologiques moyennement instables, en milieu nuageux et sous des précipitations faibles. Son principe de pilotage est simple : pour monter, on jette un peu de lest (sable) ; une cuillère à café de sable suffit pour s'élever de plusieurs dizaines de mètres ; pour descendre, on laisse s'échapper un peu d'hélium par une soupape ; enfin, pour atterrir, le pilote fait sortir complètement I'hélium de l'enveloppe.

L'enveloppe du ballon à air chaud ou montgolfière comprend plusieurs fuseaux confectionnés dans du nylon très léger, étanche et ininflammable. Pour réchauffer l'air atmosphérique contenu dans cette enveloppe, on utilise un puissant brûleur au propane. Au sommet de l'enveloppe, une soupape commandée par le pilote permet à l'air chaud de s'évacuer. Les ascensions et les vols ne peuvent se faire qu'en présence de masses d'air stables : quand les planeurs volent, les montgolfières restent au sol. Pour s'élever, le pilote chauffe l'air contenu dans l'enveloppe. L'air ainsi réchauffé se dilate et fait " gonfler » l'enveloppe. La montgolfière ne décolle pas tant que la température de l'air au sein de l'enveloppe reste inférieure à celle de l'air ambiant. Lorsque ces deux températures sont égales, la montgolfière est en équilibre à un certain niveau dans l'atmosphère : c'est le vol en palier. La descente s'amorce lorsque la température de l'air de l'enveloppe devient inférieure à celle de l'air extérieur.

Le ballon à gaz et la montgolfière sont les esclaves du vent, avec ses caprices, ses changements de direction et de vitesse. Comme des bulles de savon, ils "flottent " au gré des mouvements de l'air dans l'atmosphère. 


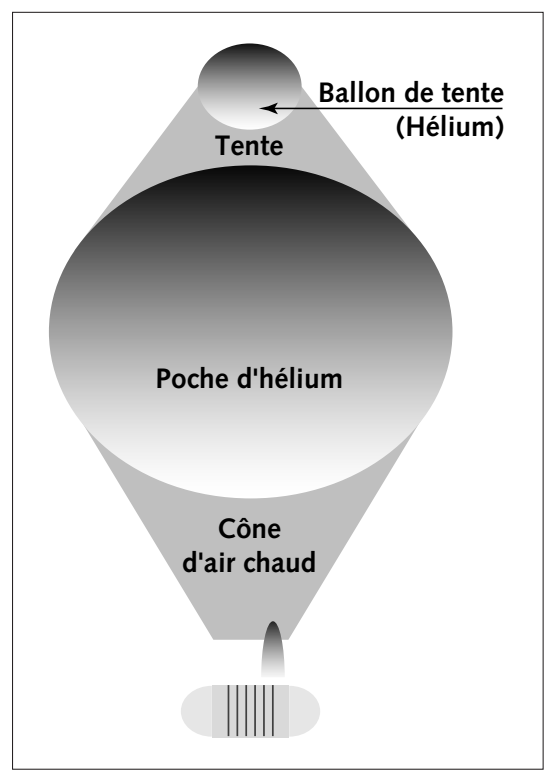

Figure 1 - Schéma de principe du ballon du Breitling Orbiter III. (@ Breitling)

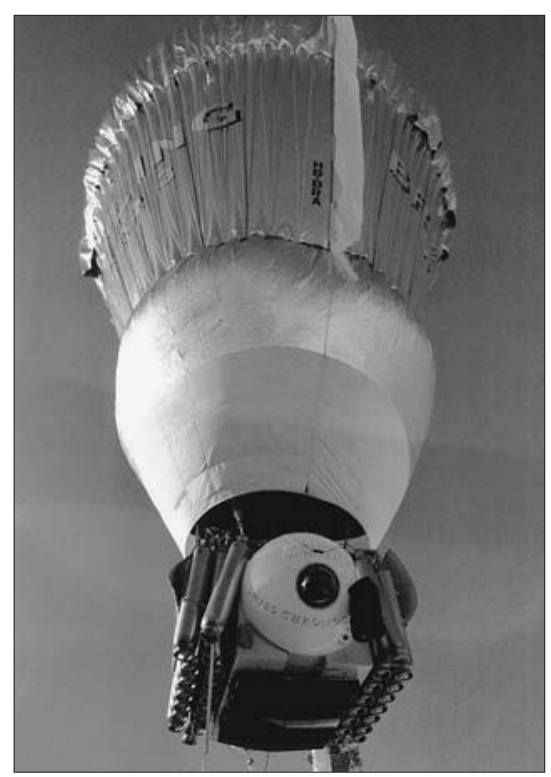

La cabine pressurisée et les réservoirs de propane du Breitling Orbiter III, peu après le décollage, le $1^{\text {er }}$ mars 1999. (@ AFP Photo, Fabrice Coffrini).

\section{Les brûleurs au propane}

\author{
La cabine pressurisée
}

Le chauffage de l'air de la partie «montgolfière » du Breitling Orbiter III est assuré par des brûleurs au propane. Le propane est stocké dans 28 réservoirs de 2,35 mètres de haut et de $100 \mathrm{~kg}$ chacun, qui sont disposés de part et d'autre de la cabine pressurisée.

$\mathrm{Vu}$ les basses températures et la raréfaction de l'oxygène rencontrées aux altitudes où vole le ballon, la présence d'une cabine pressurisée permet aux deux hommes d'équipage de travailler dans des conditions correctes. Accrochée sous le ballon, cette cabine est construite en kevlar et en fibre de carbone. Elle mesure 5,4 mètres de long et 2,85 mètres de haut. L'alimentation électrique est assurée par cinq batteries au plomb rechargées par des panneaux solaires suspendus sous la cabine.

Dans la cabine, l'équipage dispose de tous les moyens modernes de navigation (transpondeurs radar, systèmes GPS) et de communication (radios VHF et $\mathrm{HF}$, communication satellitaire via Inmarsat, balise de détresse).

Pourquoi vouloir boucler le tour du monde le plus rapidement possible ? Tout d'abord à cause de l'autonomie permise par les stocks de propane et limitée à 21 jours pour le Breitling Orbiter III. Ensuite parce que cela fait partie de la performance, du record.

Pour pouvoir se déplacer le plus rapidement possible, il faut profiter des vents forts, et plus particulièrement des courants-jets, ces courants rapides que l'on trouve aux altitudes élevées de la troposphère. Piloter un ballon, c'est donc jouer avec les lois de la physique, en fonction de l'état actuel et prévu de l'atmosphère. On mesure ainsi toute l'importance de l'assistance et du routage météorologiques.

$\mathrm{Au}$ vu des analyses et des prévisions fournies par les modèles numériques, les météorologistes-routeurs doivent donc conseiller aux pilotes du ballon l'altitude de vol la plus judicieuse pour profiter des vents favorables : si le courant-jet est bien établi, faire en sorte de demeurer à l'intérieur ; s'il s'affaiblit, le quitter en descendant pour exploiter les vents de basse ou moyenne altitude ; aller chercher un courant-jet naissant, etc. En cela, l'art du routage d'un ballon est assez proche de celui du routage des voiliers en mer; néanmoins, alors que le voilier se déplace au niveau de la mer, dans deux dimensions, le ballon se meut dans les trois dimensions et la verticale acquiert dans ce cas une importance capitale. 


\section{Les courants-jets}

Découverts par des pilotes américains le 24 novembre 1944, lors d'un raid sur Tokyo, les courants-jets sont des tubes de vents forts dans lesquels on peut observer des vitesses supérieures à $300 \mathrm{~km} / \mathrm{h}$. La zone de vent fort peut avoir plusieurs centaines de kilomètres de large et 2000 à 3000 mètres d'épaisseur (figure 2). Ces courants-jets sont généralement observés dans la haute troposphère ( 6 à $12 \mathrm{~km}$ d'altitude). Les avions de ligne tiennent compte de la présence de ces courants-jets pour choisir leur route et économiser du carburant.

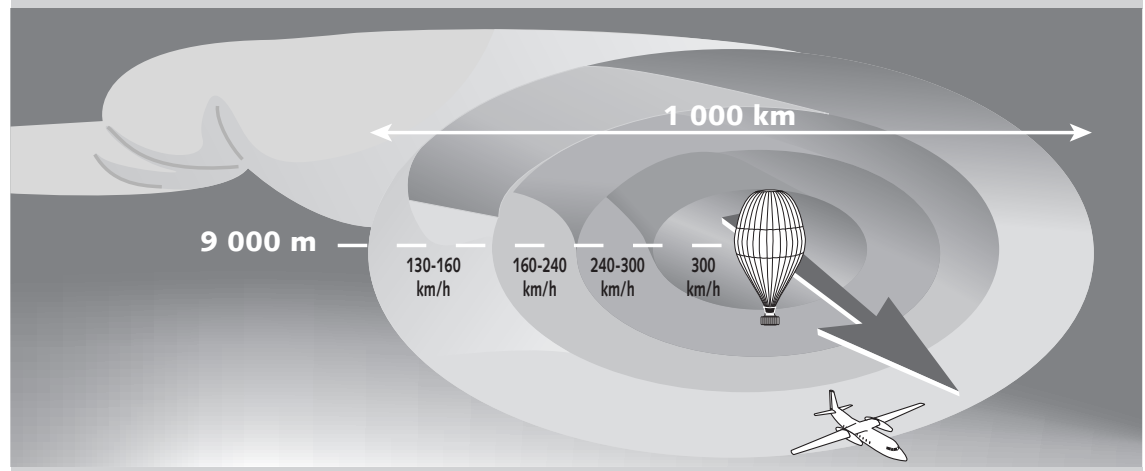

Figure 2 - Schéma simplifié d'un courant-jet. (๑) Breitling)

Dans l'hémisphère nord, les principaux courants-jets sont :

- Le jet polaire situé aux latitudes tempérées avec des vents d'ouest. Non permanent, il est marqué par de fortes variations dans le temps et l'espace.

- Le jet subtropical (vents d'ouest) situé au voisinage du $30^{\circ}$ nord. II est quasi permanent et suit le mouvement apparent du soleil (position la plus au nord en mars et position la plus au sud en septembre). Les valeurs maximales de vitesse du vent sont observées lors de la période de janvier à mars. Ce jet est une réalité climatologique, comme le montre la carte moyenne du vent à $200 \mathrm{hPa}$ au mois de janvier (figure 3 ).

- Le jet équatorial d'est (vents d'est) qui souffle sur le $12^{\circ}$ nord, du sud des Philippines aux côtes occidentales de l'Afrique. II a pour origine le fort gradient thermique horizontal existant dans la moyenne troposphère (5 $000-7000 \mathrm{~m}$ ) entre le continent asiatique (zone chaude) et les régions équatoriales (zone froide).

Dans certaines situations météorologiques, le jet polaire et le jet subtropical peuvent se rejoindre, avec pour conséquence principale une circulation de vents forts tout autour de la Terre et l'apparition de temps très perturbé dans les basses couches.

Figure 3 - Carte climatologique du vent à $200 \mathrm{hPa}$ au mois de janvier. Cette carte est une moyenne sur quatorze années (1979-1992) des réanalyses correspondantes du CEPMMT, interpolée sur une grille d'environ 3,75 × 3,75 degrés. Elle comporte des flèches indiquant la direction et la vitesse

du vent (la flèche sous la figure correspond à $50 \mathrm{~m} / \mathrm{s}$, soit $180 \mathrm{~km} / \mathrm{h}$ ). Sont aussi représentées en niveaux de gris les zones iso-module du vent (tous les $10 \mathrm{~m} / \mathrm{s}$ )

On observe la présence " climatologique " du courant-jet subtropical vers $30^{\circ}$ nord grosso modo entre 0 et $180^{\circ}$ est.

(Document Météo-France, Centre national de recherches météorologiques)

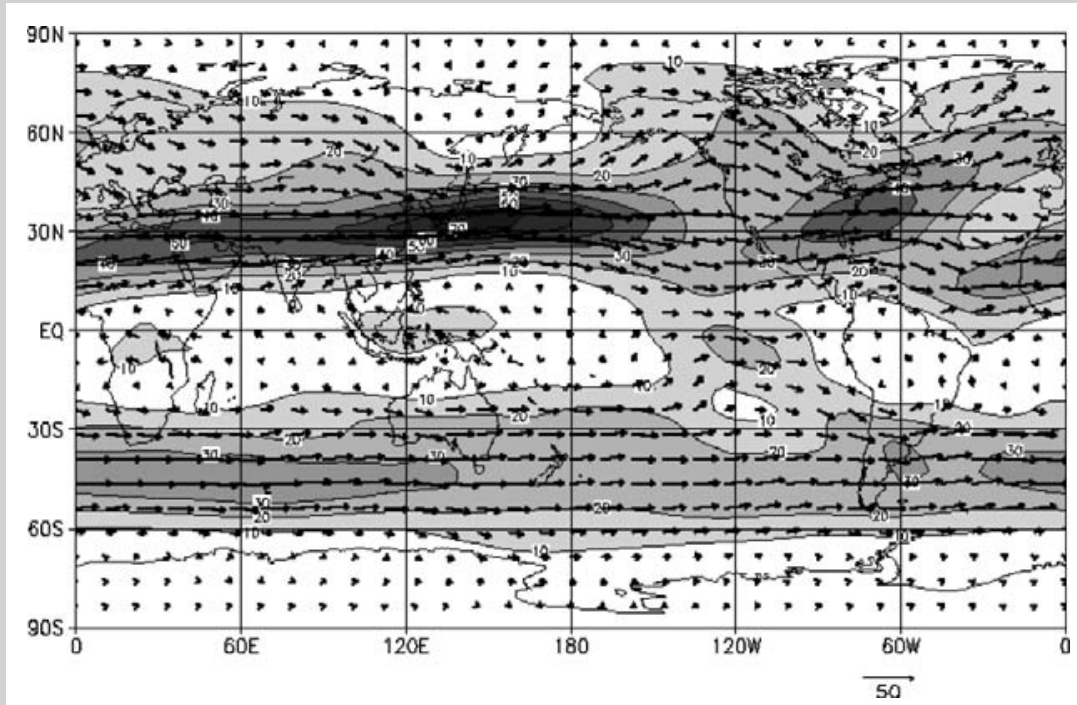




\section{Quelques situations rencontrées lors du tour du monde}

Le routage ne se limite pas à la recherche des vents les plus rapides. En effet, à tout moment, des variations infimes de nébulosité, d'ensoleillement, de direction ou de vitesse du vent peuvent contrecarrer la bonne progression du ballon. $\mathrm{Si}$, par exemple, au cours d'une phase de vol en palier, le rayonnement solaire s'accroît, l'hélium se réchauffe, se dilate, et la force ascensionnelle augmente brutalement. Le ballon et son équipage se retrouvent alors à un niveau supérieur, où peut-être la direction et la vitesse du vent ne sont plus du tout favorables. Au contraire, une forte nébulosité provoque un refroidissement de l'hélium et le ballon descend, avec d'autres conditions et contraintes atmosphériques. On comprend donc que les routeurs doivent prévoir l'emplacement des zones nuageuses d'altitude élevée.

Les routeurs doivent aussi aider les pilotes du ballon à éviter les dangers que recèle l'atmosphère : la turbulence qui agite le ballon ; l'humidité, les nuages, les précipitations et le givrage qui alourdissent l'enveloppe et diminuent l'autonomie de vol ; enfin, les orages dont les violents courants verticaux peuvent déchirer l'enveloppe.

La figure 4 montre le parcours horizontal du Breitling Orbiter III ; on observe qu'une fois arrivé dans la zone tropicale (point D), le ballon s'est toujours maintenu entre 8 et 28 degrés de latitude nord ${ }^{(1)}$. Les lettres inscrites sur la carte mentionnent différents pointages, dont les dates et heures sont données dans le tableau 1. On notera que le trajet F-G-H correspond à une inflexion du parcours du ballon vers le nord, afin de contourner une zone militaire dont le survol a été interdit par le Yémen.

Le tableau 2 donne des renseignements plus détaillés sur le parcours du Breitling Orbiter III : altitude et vitesse moyennes du ballon pour les différents segments de son trajet. Les indications JS1, JS2 et JS3 portées dans ce tableau correspondent aux entrées du ballon dans les courants-jets : dans le Sud de la Chine le 9 mars pour JS1, au sud-est d'Hawaii le 15 mars pour JS2, enfin sur l'Atlantique le 19 mars pour JS3. Ces situations à courant-jet ont été, bien entendu, les plus favorables pour la réussite du tour du monde. À d'autres moments, au contraire, le ballon a volé très lentement faute d'un vent suffisant : cela a été notamment le cas à l'arrivée au-dessus du Pacifique (points K, L et M), avec une « mise en attente » du Breitling Orbiter III avant le choix d'une route vers le nord-est ou vers le sud-est. Au point M, le 12 mars, c'est finalement la route vers le sud-est qui sera choisie.

(1) Pour que le tour du monde soit homologué par la Fédération aéronautique internationale, il faut qu'il soit effectué à plus de 30 degrés de latitude des pôles.

Figure 4 - Le parcours du Breitling Orbiter III. Les lettres correspondent à différents pointages successifs, dont les dates et heures sont données dans le tableau 1.

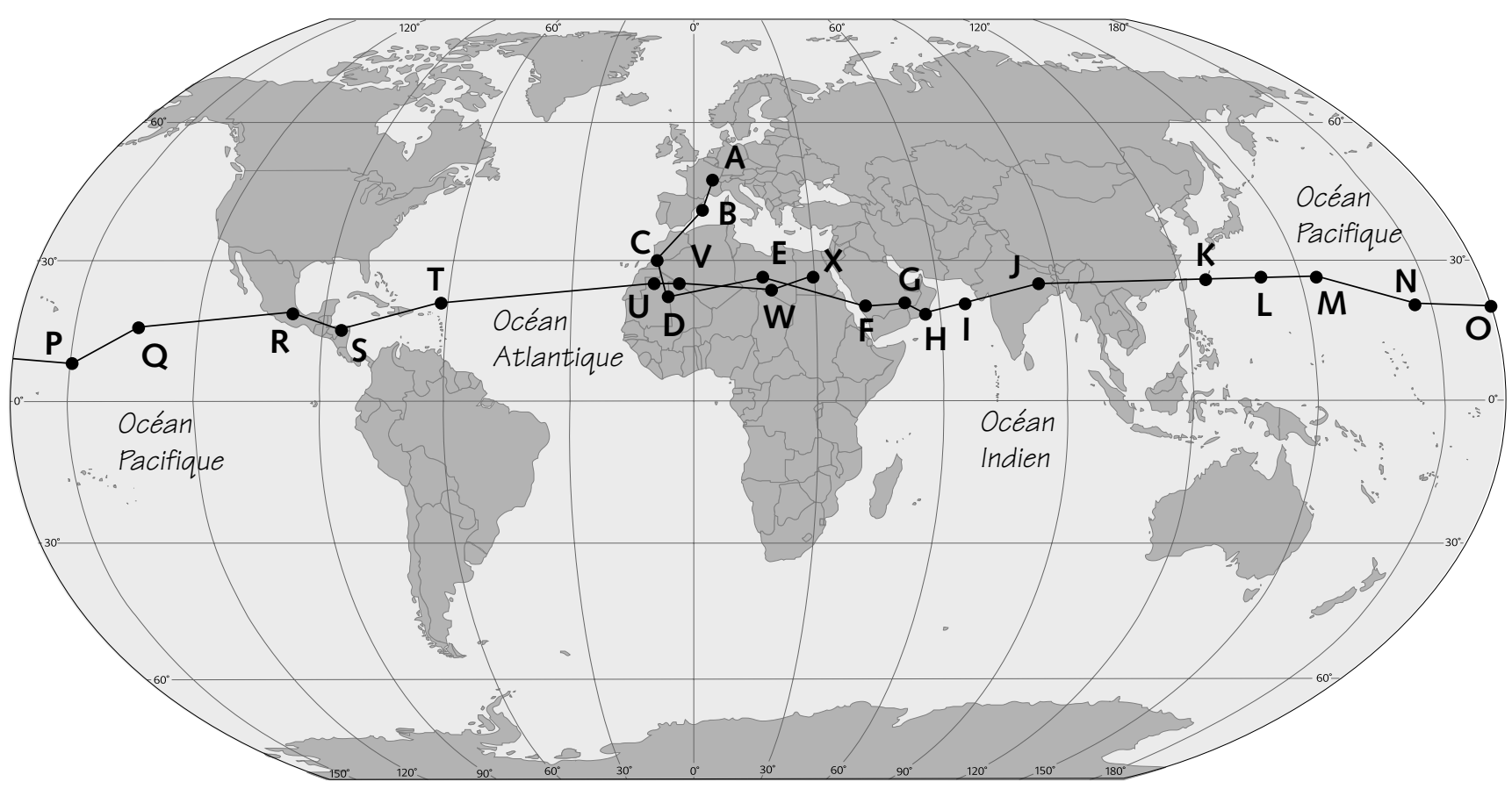


Tableau 1 - Date et heure de passage aux points mentionnés sur la figure 4.

Tableau 2 - Altitude et vitesse moyennes du Breitling Orbiter III pour les différents segments de son parcours. Les entrées dans les courants-jets sont repérées par JS1, JS2 et JS3. Les zones ombrées renvoient aux cartes météorologiques des figures 5 et 6 .

\begin{tabular}{|c|c|c|c|}
\hline $\begin{array}{c}\text { Point } \\
\text { du } \\
\text { parcours }\end{array}$ & Date & $\begin{array}{c}\text { Heure } \\
\text { UTC }\end{array}$ & \\
\hline A & 1 mars & 8 h 05 & Départ \\
\hline B & 2 mars & $8 \mathrm{~h}$ & \\
\hline C & 3 mars & $7 \mathrm{~h}$ & \\
\hline D & 4 mars & $7 \mathrm{~h}$ & \\
\hline$E$ & 5 mars & $8 \mathrm{~h}$ & \\
\hline $\mathbf{F}$ & 6 mars & $13 \mathrm{~h}$ & Contournement du Yémen \\
\hline G & 6 mars & $22 \mathrm{~h}$ & Contournement du Yémen \\
\hline $\mathbf{H}$ & 7 mars & $11 \mathrm{~h}$ & \\
\hline I & 8 mars & $8 \mathrm{~h}$ & \\
\hline J & 9 mars & $7 \mathrm{~h}$ & \\
\hline K & 10 mars & $18 \mathrm{~h}$ & \\
\hline $\mathbf{L}$ & 11 mars & $8 \mathrm{~h}$ & \\
\hline$M$ & 12 mars & $6 \mathrm{~h}$ & \\
\hline $\mathrm{N}$ & 13 mars & $10 \mathrm{~h}$ & \\
\hline O & 14 mars & $7 \mathrm{~h}$ & \\
\hline $\mathbf{P}$ & 15 mars & $7 \mathrm{~h}$ & \\
\hline Q & 16 mars & $11 \mathrm{~h}$ & \\
\hline $\mathbf{R}$ & 17 mars & $11 \mathrm{~h}$ & \\
\hline$S$ & 18 mars & $7 \mathrm{~h}$ & \\
\hline$T$ & 19 mars & $8 \mathrm{~h}$ & \\
\hline U & 20 mars & 9 h 54 & Passage de la ligne d'arrivée \\
\hline V & 20 mars & $12 \mathrm{~h}$ & \\
\hline W & 21 mars & $0 \mathrm{~h}$ & \\
\hline$x$ & 21 mars & $6 \mathrm{~h} 03$ & Atterrissage \\
\hline
\end{tabular}

\begin{tabular}{|c|c|c|c|c|c|}
\hline & & $\begin{array}{l}\text { Altitude } \\
\text { moyenne }\end{array}$ & $\begin{array}{l}\text { Vitesse } \\
\text { moyenne } \\
\text { (nœuds) }\end{array}$ & $\begin{array}{l}\text { Vitesse } \\
\text { moyenne } \\
(\mathrm{km} / \mathrm{h})\end{array}$ & \\
\hline$A$ & B & 6800 & 20 & 37 & \\
\hline B & $\mathrm{C}$ & 7000 & 40 & 74 & \\
\hline $\mathrm{C}$ & D & 5500 & 25 & 46 & \\
\hline D & $E$ & 8000 & 60 & 111 & \\
\hline$E$ & $\mathbf{F}$ & 6700 & 65 & 120 & \\
\hline $\mathbf{F}$ & G & 5600 & 50 & 93 & \\
\hline G & $\mathrm{H}$ & 3500 & 30 & 56 & \\
\hline $\mathrm{H}$ & I & 5000 & 40 & 74 & \\
\hline I & J & 7000 & 40 & 74 & \\
\hline$J$ & $\mathrm{~K}$ & 8800 & $70-90$ & $129-167$ & JS1 \\
\hline$K$ & $M$ & 7600 & 40 & 74 & \\
\hline$M$ & $\mathrm{O}$ & 7500 & 40 & 74 & \\
\hline 0 & $\mathbf{P}$ & 9500 & 40 & 74 & \\
\hline $\mathbf{P}$ & $\mathrm{Q}$ & 9500 & 70 & 130 & JS2 \\
\hline$Q$ & $\mathbf{R}$ & 10000 & 100 & 185 & \\
\hline $\mathbf{R}$ & $S$ & 10500 & 45 & 83 & Figure 5 \\
\hline$S$ & $\mathrm{~T}$ & 11000 & 60 & 111 & \\
\hline$T$ & $U$ & 11000 & 90 & 167 & JS3 \\
\hline$U$ & V & 11000 & 90 & 167 & \\
\hline V & W & 11500 & 120 & 222 & Figure 6 \\
\hline W & $x$ & Atterrissage & 5 & 9 & \\
\hline
\end{tabular}


Figure 5 - Situation météorologique observée à $250 \mathrm{hPa}$, le 17 mars 1999 à $12 \mathrm{~h}$ UTC, au-dessus de I'Amérique centrale. Le vent en altitude est représenté, d'une part par les lignes de courant qui donnent en tout point sa direction, de l'autre par les niveaux de gris qui donnent le module du vent en nœuds. La position du Breitling Orbiter III est indiquée par le carré blanc. On observe que le courant-jet subtropical (vent d'ouest supérieur à 60 nœuds, soit $110 \mathrm{~km} / \mathrm{h}$ ) est fractionné en deux parties, l'une sur le Pacifique est, l'autre sur l'Atlantique ouest. (C) NOAA et Luc Trullemans)
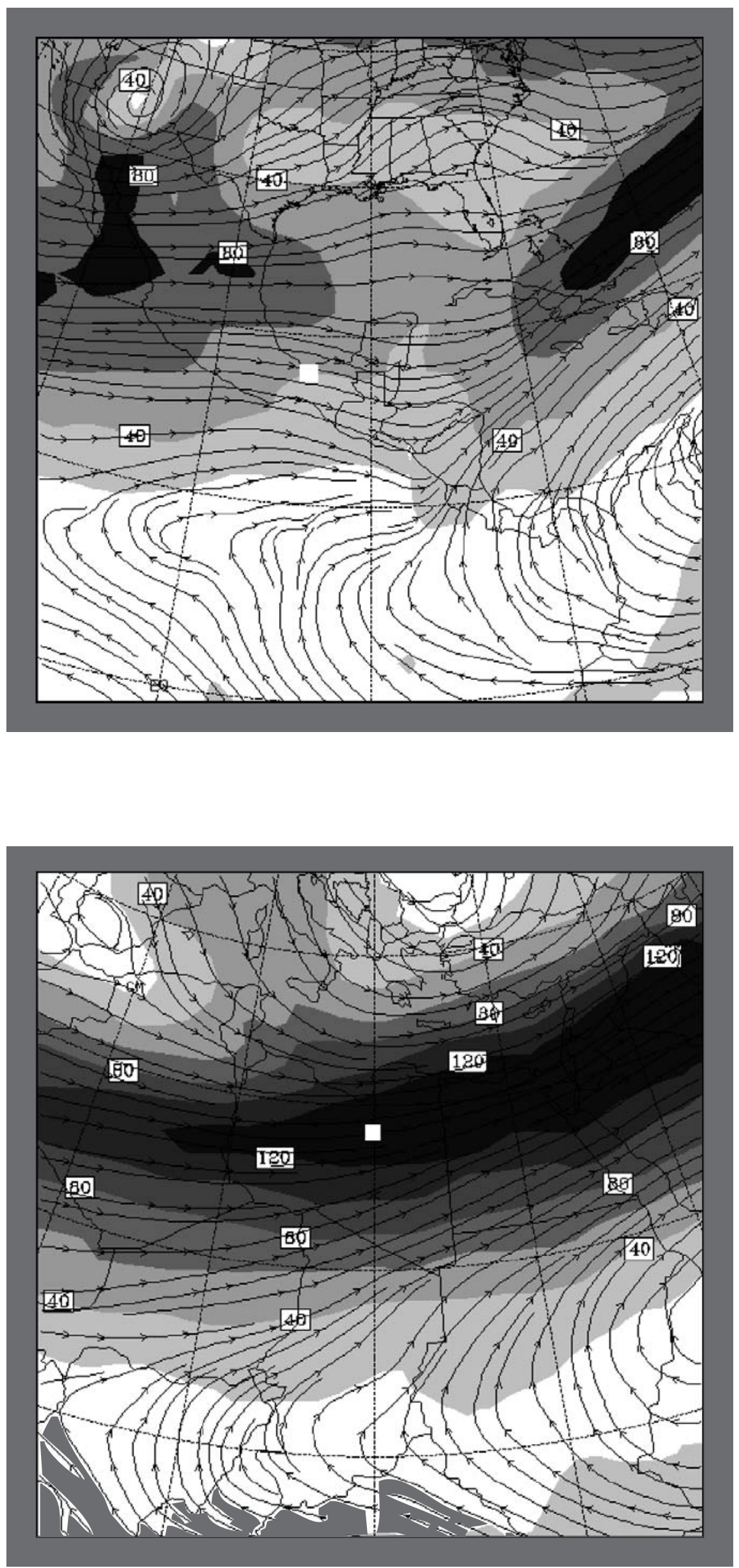

Figure 6 - Situation météorologique observée à $200 \mathrm{hPa}$, le 21 mars 1999 à $0 \mathrm{~h}$ UTC, au-dessus du Nord de I'Afrique. La représentation est similaire à celle de la figure 5 . Dans ce cas, le courant-jet subtropical est présent sur tout le domaine, avec des vents d'ouest dépassant largement les 120 nœuds $(220 \mathrm{~km} / \mathrm{h})$ dans la région où se trouve le ballon. (@ NOAA et Luc Trullemans) 


\section{7 mars, situation à vent faible}

Nous avons choisi d'illustrer la « météo du tour du monde » à l'aide de deux situations en altitude très différentes, l'une à vent faible, l'autre à courant-jet, repérées par les zones ombrées sur le tableau 2.

Le 17 mars à 11 h UTC, le Breitling Orbiter III est au-dessus de l'Amérique centrale (point $\mathrm{R}$ ). Après une deuxième moitié de traversée du Pacifique très rapide grâce à un courant-jet (JS2), sa vitesse se réduit à environ $75 \mathrm{~km} / \mathrm{h}$ (altitude proche de $11000 \mathrm{~m}$ ). La figure 5 montre la situation météorologique à $250 \mathrm{hPa}$, le 17 mars à $12 \mathrm{~h}$ UTC ; les traits fins sont les lignes de courant parallèles à la direction du vent ; les zones grisées correspondent à un module du vent supérieur à 60 kt (environ $110 \mathrm{~km} / \mathrm{h}$ ). La position du ballon est indiquée par un carré blanc. On observe un courant-jet fractionné en deux parties : l'une sur le Pacifique est, l'autre sur l'Atlantique ouest. Entre les deux, et dans la région où se trouve le ballon, le vent est d'ouest, plutôt faible (environ $40 \mathrm{kt}$ à l'endroit où se trouve le ballon). Cette journée du 17 mars sera d'ailleurs l'une des plus pénibles pour Piccard et Jones ; citons la rubrique « En direct » de ce jour-là, affichée sur le site Internet du Breitling Orbiter III : « Lors d'un bref entretien avec le centre de contrôle, Bertrand Piccard et Brian Jones se sont avoué très fatigués par cette fin de trajet. Le ballon est sorti plus tôt que prévu du courant-jet et a ainsi pris une direction plus au sud qu'initialement envisagé. À bord, les aérostiers vivent des moments difficiles, la température intérieure de la capsule à 11000 mètres n'étant que de $8^{\circ} \mathrm{C}$. »
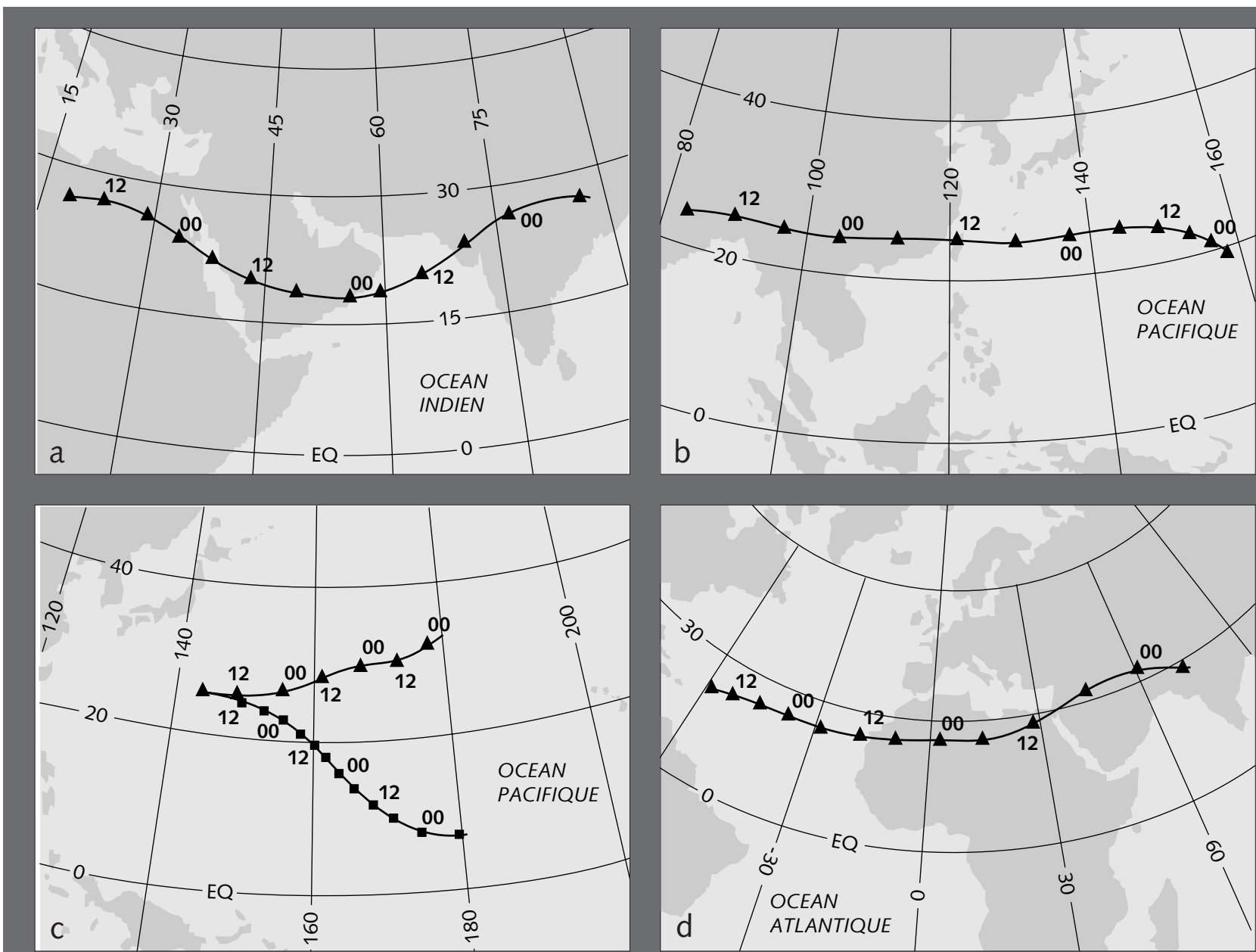

Figure 7 - Cartes de trajectoires prévues établies par Luc Trullemans à différents moments du tour du monde en ballon. Chaque petit segment correspond à un intervalle de 6 heures. La trajectoire complète correspond à 3 jours.

a - trajectoire commençant le 5 mars 1999 à 8 h UTC, au niveau $450 \mathrm{hPa}$; b - trajectoire commençant le 9 mars 1999 à 7 h UTC, au niveau $350 \mathrm{hPa}$; c - trajectoires commençant le 11 mars 1999 à $8 \mathrm{~h} \mathrm{UTC}$, aux niveaux $400 \mathrm{hPa}$ (triangles) et $300 \mathrm{hPa}$ (carrés) ; d - trajectoire commençant le 19 mars 1999, à $8 \mathrm{~h}$ UTC, au niveau $250 \mathrm{hPa}$. 
21 mars, situation à courant-jet Exemples
de trajectoires prévues

POUR CONCLURE
Le 21 mars à 0 h UTC, la situation est bien meilleure pour Piccard et Jones : d'abord, depuis le passage au-dessus de la Mauritanie, le tour du monde est officiellement bouclé ; ensuite, alors que le Breitling Orbiter III se trouve au-dessus de la Libye (point $\mathrm{W}$ ), à près de $11500 \mathrm{~m}$ d'altitude, la situation météo est plus favorable que jamais (figure 6 , à $200 \mathrm{hPa}$ ). Le courant-jet est cette fois bien établi, avec autour du ballon des vents d'ouest à environ $130 \mathrm{kt}$, soit $240 \mathrm{~km} / \mathrm{h}$. L'Égypte n'est plus très loin et l'atterrissage est pour dans six heures !

La figure 7 montre quatre exemples de trajectoires prévues par Luc Trullemans lors du tour du monde, avec pour points de départ et pour niveaux de pression respectifs : le 5 mars à $8 \mathrm{~h} \mathrm{UTC}, 450 \mathrm{hPa}$ (figure 7a); le 9 mars à $7 \mathrm{~h} \mathrm{UTC}, 350 \mathrm{hPa}$ (figure 7b) ; le 11 mars à $8 \mathrm{~h} \mathrm{UTC}, 300$ et $400 \mathrm{hPa}$ (figure 7c) ; le 19 mars à 8 h UTC, $250 \mathrm{hPa}$ (figure 7d).

En comparant ces trajectoires prévues avec la trajectoire réelle de la figure 4, on observera que la concordance générale est excellente. On notera également sur la figure $7 \mathrm{c}$, établie alors que le ballon se trouvait au point $\mathrm{L}$, dans le Pacifique ouest, qu'il existe deux trajectoires possibles : l'une vers le nord-est à $400 \mathrm{hPa}$, l'autre vers le sud-est à $300 \mathrm{hPa}$. C'est finalement la route sud-est qui a été choisie, permettant au Breitling Orbiter III d'aller rejoindre plus loin un courant-jet au sud d'Hawaii.

C'est ainsi que, 137 ans après la parution du roman prémonitoire de Jules Verne, Cinq semaines en ballon, le rêve est devenu réalité. S'il est impossible de citer ici tous les précurseurs de cet exploit moderne, on peut quand même rappeler que, le 4 juin 1783, les frères Montgolfier faisaient voler le premier ballon à air chaud et que, le 21 novembre 1783, Pilâtre de Rozier et le marquis d'Arlandes effectuaient le premier vol humain en montgolfière.

Il est probable que le tour du monde du Breitling Orbiter III n'aurait pas été possible sans les progrès récents de la météorologie. En 1992, Luc Trullemans routait, lors de la première course transatlantique en ballon, l'équipage Bertrand Piccard - Ron Cameron. Il n'utilisait alors que des prévisions numériques à 2-3 jours d'échéance. Pour le tour du monde, Luc Trullemans et Pierre Eckert se sont appuyés sur des prévisions numériques à 5-6 jours.

Ainsi, le succès de Bertrand Piccard et Brian Jones est aussi un peu celui de tous les météorologistes. Alors, ne boudons pas notre plaisir... 